\title{
Systematic vs. on-demand early palliative care in gastric cancer patients: a randomized clinical trial assessing patient and healthcare service outcomes
}

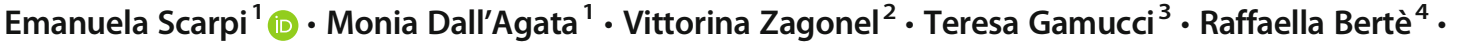 \\ Elisabetta Sansoni ${ }^{5}$. Elena Amaducci ${ }^{6}$. Chiara Maria Broglia ${ }^{7}$. Sara Alquati ${ }^{8}$ - Ferdinando Garetto ${ }^{9}$. \\ Stefania Schiavon ${ }^{2}$. Silvia Quadrini ${ }^{3}$. Elena Orlandi ${ }^{10}$. Andrea Casadei Gardini ${ }^{11}$ - Silvia Ruscelli ${ }^{11}$ - Daris Ferrari ${ }^{12}$. \\ Maria Simona Pino ${ }^{13}$. Roberto Bortolussi ${ }^{14}$. Federica Negri ${ }^{15}$. Silvia Stragliotto ${ }^{2}$. Filomena Narducci ${ }^{3}$. \\ Martina Valgiusti ${ }^{11}$. Alberto Farolfi ${ }^{11}$ - Oriana Nanni ${ }^{1} \cdot$ Romina Rossi $^{5} \cdot$ Marco Maltoni $^{5} \cdot$ on behalf of the Early \\ Palliative Care Italian Study Group (EPCISG)
}

Received: 3 May 2018 / Accepted: 16 October 2018

(C) Springer-Verlag GmbH Germany, part of Springer Nature 2018

\begin{abstract}
Purpose Early palliative care (EPC) has shown a positive impact on quality of life (QoL), quality of care, and healthcare costs. We evaluated such effects in patients with advanced gastric cancer.

Methods In this prospective, multicenter study, 186 advanced gastric cancer patients were randomized 1:1 to receive standard cancer care (SCC) plus on-demand EPC (standard arm) or SCC plus systematic EPC (interventional arm). Primary outcome was a change in QoL between randomization (T0) and T1 (12 weeks after T0) in the Trial Outcome Index (TOI) scores evaluated through the Functional Assessment of Cancer Therapy-Gastric questionnaire. Secondary outcomes were patient mood, overall survival, and family satisfaction with healthcare and care aggressiveness.

Results The mean change in TOI scores from T0 to T1 was - 1.30 (standard deviation (SD) 20.01) for standard arm patients and 1.65 (SD 22.38) for the interventional group, with a difference of 2.95 (95\% CI - 4.43 to 10.32) $(p=$ 0.430). The change in mean Gastric Cancer Subscale values for the standard arm was 0.91 (SD 14.14) and 3.19 (SD $15.25)$ for the interventional group, with a difference of 2.29 (95\% CI -2.80 to 7.38$)(p=0.375)$. Forty-three percent of patients in the standard arm received EPC.

Conclusions Our results indicated a slight, albeit not significant, benefit from EPC. Findings on EPC studies may be underestimated in the event of suboptimally managed issues: type of intervention, shared decision-making process between
\end{abstract}

Emanuela Scarpi

emanuela.scarpi@irst.emr.it

1 Unit of Biostatistics and Clinical Trials, Istituto Scientifico Romagnolo per lo Studio e la Cura dei Tumori (IRST) IRCCS, Via P. Maroncelli 40, 47014 Meldola, FC, Italy

2 Medical Oncology Unit 1, Veneto Institute of Oncology IOV-IRCCS, Padova, Italy

3 Oncology Unit, SS Trinità Hospital, Sora, ASL Frosinone, Italy

4 Palliative Care, Oncology Department, Guglielmo da Saliceto Hospital, AUSL, Piacenza, Italy

5 Palliative Care Unit, Istituto Scientifico Romagnolo per lo Studio e la Cura dei, Tumori (IRST) IRCCS, Meldola, Italy

6 Palliative Care and Hospice Unit, AUSL Romagna, Cesena, Italy

7 Oncology Unit, Fondazione IRCCS, Policlinico San Matteo, Pavia, Italy
8 Palliative Care Unit, Arcispedale S. Maria Nuova-IRCCS, Reggio Emilia, Italy

9 Medical Oncology Unit, Presidio Humanitas Gradenigo, Torino, Italy

10 Medical Oncology Unit, Oncology Department, Guglielmo da Saliceto Hospital, Piacenza, Italy

11 Department of Medical Oncology, Istituto Scientifico Romagnolo per lo Studio e la Cura dei Tumori (IRST) IRCCS, Meldola, Italy

12 Oncology Unit, San Paolo Hospital, Milan, Italy

13 Medical Oncology Unit, Oncology Department, Azienda USL Toscana Centro, S. Maria Annunziata Hospital, Florence, Italy

14 Palliative care and Pain Therapy Unit, Aviano National Cancer Institute, Aviano, Italy

15 Medical Oncology Unit, Azienda Socio Sanitaria Territoriale, Cremona, Italy 
oncologists and PC physicians, risk of standard arm contamination, study duration, timeliness of assessment of primary outcomes, timeliness of cohort inception, and recruitment of patients with a significant symptom burden.

Clinical trial registration ClinicalTrials.gov (NCT01996540).

Keywords Early palliative care · Quality of life · Aggressiveness in end of life · Quality of care

\section{Introduction}

The most recent World Health Organization (WHO) definition of palliative care (PC) states that PC "is applicable early in the course of illness, in conjunction with other therapies that are intended to prolong life, such as chemotherapy or radiation therapy" [1]. PC is divided into end of life (EoL) PC and early palliative care (EPC), the latter referring to $\mathrm{PC}$ performed alongside rather than at the end of antineoplastic therapies [2]. EPC is also known as simultaneous PC [3]. Indicators of integration between oncology and PC comprise structure, process, outcome, and education [4]. An evaluation of prognosis is needed before referring patients for EoL-PC, whereas EPC requires an assessment of the PC needs of patients [5, 6]. The typical care setting for EPC is outpatient-based [7-9]. It has been suggested that the simultaneous presence of a "generalist EPC level" and a "specialist EPC level" is needed [10]. Evaluating the efficacy of EPC is difficult as there is still no single definition of EPC and of the patients who are eligible for EPC [11], i.e., the inception cohort [7]. The ideal duration of the intervention and/or the ideal time at which to examine the chosen end point are other issues that need to be addressed [7]. The integration of EPC consisting in the presence of a multidisciplinary specialist PC team is not present in all models [11]. The EPC intervention is variable in intensity, ranging from sporadic consultation, to systematic consultation, to full responsibility for care by the specialist PC group. Furthermore, the weight of PC intervention is also dependent on primary care oncologists accepting to share the decision-making process with their palliative care colleagues [12]. In such circumstances, there is a risk of contamination from the standard PC arm (crossover) and/or compensation, the latter defined as a higher level of competence in PC needs by the attending oncologists [13]. Despite this, EPC has been studied and has shown varying degrees of efficacy in outcomes concerning quality of life (QoL), quality of care, and healthcare costs [14].

We present the results from our multicenter randomized clinical trial comparing standard oncologic care plus systematic EPC with standard oncologic care plus on-demand EPC in patients with metastatic or locally advanced inoperable gastric cancer. We previously focused on the same theme in patients with pancreatic cancer $[15,16]$.

\section{Materials and methods}

\section{Study design}

This was a multicenter, randomized Italian clinical trial of standard care plus systematic early palliative care (EPC) (interventional arm) vs. standard care plus on-demand EPC (standard arm) for patients with newly diagnosed locally advanced and/or metastatic gastric cancer. The study was approved by the Ethical Committee of each participating centers and was performed in accordance with the Declaration of Helsinki.

\section{Patient selection}

Patient eligibility criteria comprised a diagnosis of inoperable locally advanced and/or metastatic gastric cancer made a maximum of 8 weeks prior to enrollment, suitability for anticancer treatment, age $\geq 18$ years, Eastern Cooperative Oncology Group (ECOG) performance status 0-2, life expectancy $>2$ months, and ability to complete questionnaires. All newly referred patients were considered for enrollment in the study. Patients were not eligible if they were already undergoing PC, had received prior chemotherapy for metastatic or advanced disease, or had already taken part in a clinical trial. All patients provided written informed consent (ClinicalTrials.gov NCT01996540).

\section{Randomization}

Consecutive eligible patients were randomized to one of the two study groups with a 1:1 allocation rate. Central randomization via fax was carried out by the Unit of Biostatistics and Clinical Trials of Istituto Scientifico Romagnolo per lo Studio e la Cura dei Tumori (IRST) IRCCS, which also was responsible for data collection and processing. Separate randomization lists were computer-generated using a variable permuted block balanced procedure for each participating center and were not made known to the investigators. No masking was involved in this open-label trial.

\section{Study treatment and procedures}

Patients assigned to the interventional arm had an appointment scheduled with a PC specialist who had a predefined checklist of issues to be addressed during the consultation [17]. The 
checklist of topics to be discussed during the visit of PC was the same as that used by Temel [17] and is reported in the original protocol. Patients met a PC physician within 2 weeks of enrollment and were seen every 2 to 4 weeks thereafter until death. In both arms, non protocol-scheduled meetings with a PC specialist were possible when needed. Moreover, each researcher had the option of using adjunctive routine tools of assessment that were not considered in the present study. PC appointments and interventions were scheduled on the basis of general PC guidelines [18].

In Italy, there is no specific specialization course in palliative care for physicians but rather a Masters Degree earned after obtaining a specialization in another area. In our study, it was not required to have formal PC training. Palliative care interventions were managed solely by physicians who, for the most part, had several years' clinical experience working fulltime in this specific area. Only a small number of physicians had formal training in palliative care. The full-time PC specialist who regularly saw interventional arm patients could prescribe drugs and request other interventions pertaining to physical, psychological, and spiritual needs. However, recommendations made by the PC physician on the decision-making process were required to be shared with the oncologist. Patients assigned to the standard arm were not scheduled to meet a PC physician unless they, their families, or the attending oncologist requested an appointment. These patients did not crossover to the interventional group when they underwent PC.

Informed consent was obtained at T0 (date of randomization) and patients were asked to complete QoL and mood questionnaires. At T1 (12 \pm 3 weeks after T0), information on ECOG performance status, QoL, mood, and family satisfaction with care was recorded. After T1, patients were followed by the PC physicians as needed. Following the patient's death (T2), information on the use of healthcare services and EoL care, anticancer therapy, and date and location of death were retrieved form medical records or proxy reports.

\section{Measures}

Health-related QoL and physical symptoms were measured using the Functional Assessment of Cancer Therapy-Gastric (FACT-Ga) scale [19] which assesses generic QoL concerns (physical, social, emotional, and functional well-being) and disease-specific issues (Gastric Cancer Subscale (GaCS)). FACT-Ga scores ranged from 0 to 184 and GaCS scores ranged from 0 to 76 (the higher the score, the better the QoL). The Trial Outcome Index (TOI), which combines the scores of physical, functional, and disease-specific subscales, was used to obtain an index of physical/functional outcomes. Scores ranged from 0 to 132 . Mood was assessed using the Hospital Anxiety and Depression Scale (HADS), a 14-item instrument composed of 2 subscales that screens for symptoms of anxiety and depression (scores ranged from 0 to 21 , a higher score indicating greater anxiety or depression and a score $>7$ indicating borderline or clinical anxiety or depression) [20].

Family satisfaction with care was evaluated by the Italian version of the Family Satisfaction with the End-of-Life Care (FAMCARE) questionnaire. The FAMCARE is a 20 -item scale that includes 4 subscales: information giving, physical patient care, psychosocial care, and availability of care. Scores ranged from 20 to 100 (the lower the score, the higher the family satisfaction) [21]. A caregiver was considered as "the individual identified by the patient as the person most involved in the care of the patient" [22]. Licenses to use the Italian versions of the FACT-Ga and HADS questionnaires were obtained. All questionnaires were administered by study staff.

\section{Statistical analysis}

The primary objective of the study was to compare the impact of systematic EPC with on-demand EPC during standard cancer care on QoL and clinical symptoms. Evaluation of the TOI score was the primary endpoint. Secondary objectives assessed were relief of symptom burden, mood, family satisfaction with care, use of healthcare services, location of death, and overall survival (OS). The study was designed to enroll 240 patients with advanced gastric or pancreatic cancer who were candidates for antitumor treatment. This paper presents the clinical findings relating to the gastric cancer population; the results for the pancreatic cancer group having been described elsewhere $[15,16]$. We estimated that the recruitment of 120 evaluable gastric cancer patients would give the study $80 \%$ power to detect a significant between-group difference in the change in the TOI score between $\mathrm{T} 0$ and $\mathrm{T} 1$, with an effect size of 0.5 of the standard deviation (SD). Estimating that patients would have an expected survival of $80 \%$ at $\mathrm{T} 1$ and assuming an overall dropout rate of $20 \%$, we randomized 186 patients to achieve the target sample size for the $\mathrm{T} 1$ analysis.

We computed effect sizes such as Cohen's d statistic, and an effect size of at least 0.3 was considered clinically relevant. Continuous variables were summarized by descriptive statistics (mean, SD, median, minimum and maximum), and categorical variables were summarized using counts of patients and percentages. Differences in clinical outcomes between study groups were assessed with the chi-square test for categorical variables and the Student's $t$ test or nonparametric ranking statistic (median test) for continuous variables. Logistic regression analyses were performed, adjusting for baseline characteristics (age, gender, marital status, and performance status) to examine the impact of EPC on the use of chemotherapy and on healthcare measures. OS was defined as the time from the date of randomization to the date of death due to any cause. Patients who were still alive at the time of 
analysis (October 2017) were censored at their last date of follow-up. OS was estimated with the Kaplan-Meier product-limit method and corresponding $95 \%$ confidence intervals (CI) were calculated using the Greenwood method. The statistical analysis of the primary outcome (the change in the TOI score between $\mathrm{T} 0$ and $\mathrm{T} 1$ ) was performed using the multiple imputation method to handle missing data and achieve valid statistical inference [23]. All analyses were performed on an intention-to-treat population meeting eligibility criteria, adjusted for baseline values. All tests were two-sided at a significance level of 0.05 . No interim analysis was planned and no multiplicity test correction was performed. All statistical analyses were performed using the SAS Statistical Software version 9.4 (SAS Institute, Cary, NC, USA).

\section{Results}

In this study, 186 outpatients with metastatic or locally advanced not operable gastric cancer were randomized from 21 Italian centers between August 2012 and July 2016. One hundred and seventy-nine $(96.2 \%)$ patients were eligible and evaluable at T0 (89 in the standard arm, 90 in the interventional arm). Six standard arm patients were excluded from the analysis because chemotherapy was not subsequently administered, and one patient in the interventional arm was not eligible because of prior chemotherapy (Fig. 1). Baseline characteristics of patients enrolled in both arms were superimposable (Table 1). The mean number (SD) of palliative care visits during the study period was 0.53 (1.14) for the standard arm and 4.28 (1.87) for the interventional arm, with a significantly higher number for the latter $(p<0.0001)$.

Of the179 patients evaluable at T0, 48 (26.8\%) did not complete the QoL and mood questionnaires at T1 because they were too ill or had died. The mean change in TOI scores from baseline to week 12 was - 1.30 (SD 20.01) for standard arm patients, and 1.65 (SD 22.38) for the interventional arm, with a difference between groups of 2.95 (95\% CI -4.43 to $10.32)$ in favor of the latter $(p=0.430)$ (Table 2). With respect to the GaCS score, the change in mean values was 0.91 (SD 14.14) for the standard arm and 3.19 (SD 15.25) for the interventional arm, with a difference between the two groups of

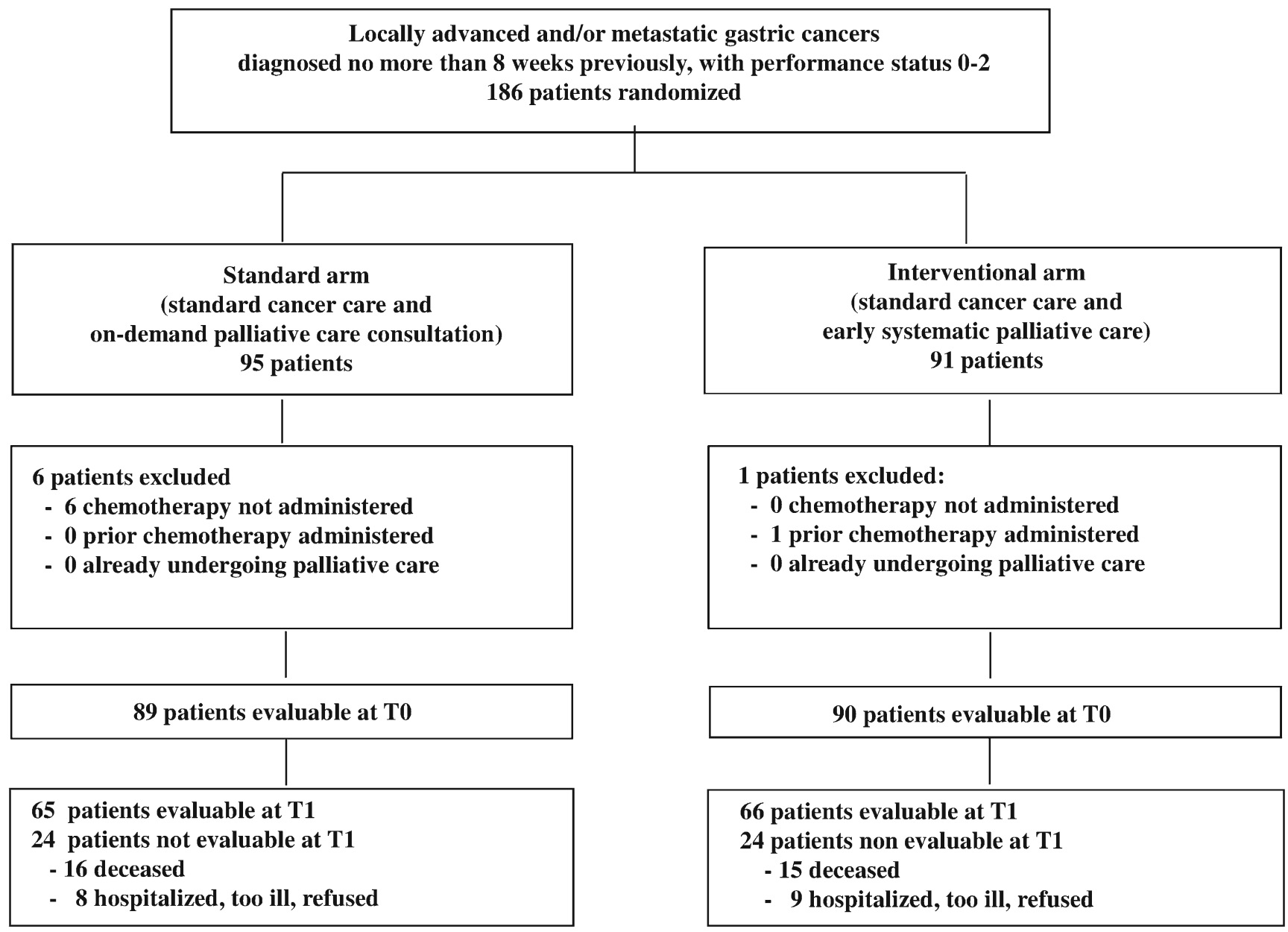

T0, baseline

$\mathrm{T} 1,12 \pm 3$ weeks after $\mathrm{T} 0$

Fig. 1 Consort diagram of the study: patient recruitment, treatment, and analysis 
$2.29(95 \% \mathrm{CI}-2.80$ to 7.38$)(p=0.375)$. Mean values of FACT-G, FACT-Ga, GaCS, and TOI at T1 were slightly higher for interventional arm patients, with $p$ values adjusted for baseline QoL scores ranging from 0.167 to 0.585 (Table 3). In a responder's analysis we performed based on clinically important changes in the TOI primary outcome, the percentage of responders was $39.4 \%$ in the intervention group and $34.8 \%$ in the standard group ( $p=0.590)$.

There were no differences between arms in the data regarding mood, HADS anxiety and depression subscales, and family satisfaction with care (data not shown).

When the results of the study were evaluated (October 2017), 149 (83.2\%) of the 179 evaluable patients had already died. Median OS was 9.9 months (95\% CI 8.4-11.5) in the standard arm and 10.2 (95\% CI 7.8-12.3) in the interventional arm, with a 12-month OS probability of $37.9 \%$ (95\% CI $27.7-$ $48.1)$ for the former and $41.3 \%(95 \%$ CI $31.0-51.7)$ for the latter $(p=0.657)$. A moderately high percentage of patients $(43 \%)$ in the standard arm received early palliative care, with a median OS of 10.0 months (95\% CI 7.4-11.8) and a median interval of 4.0 months from the first palliative care referral to death (95\% CI 1.9-6.8). Standard arm patients who did not undergo palliative care showed a median OS of 9.5 months (95\% CI 7.0-12.3).

We also evaluated some indicators of aggressiveness of care near the end of life. No significant differences were observed for the following items: chemotherapy in the last days of life, number and timeliness of hospice care activations, and place of death (Table 4).

\section{Discussion}

Data on the efficacy and efficiency of EPC have become progressively more robust over time. However, conducting studies in this palliative care setting is not easy and some negative studies have been published. Temel et al. reported improved QoL (primary outcome) and mood and survival after 12 weeks of EPC in 151 patients with lung cancer [17]. Interestingly, a more recent study by the same group did not obtain significant positive data on QoL after 12 weeks (primary outcome) in a mixed group of 350 patients with lung or gastrointestinal cancer, but rather after 24 weeks (secondary endpoint) [24]. Considered separately, significant results were obtained after both 24 and 12 weeks for the lung cancer subgroup, while gastrointestinal cancer patients did not show positive data at either of the evaluations because of higher "spontaneous" good results of the conventional group. The authors concluded that the effect of EPC was probably dependent on the specific needs of each patient population. The Zimmermann cluster randomized trial studied EPC in 461 patients with miscellaneous cancer [25]. After 3 months, the primary endpoint showed a nonsignificant trend in favor of the interventional group, with some substantially changed secondary outcome scores. After 4 months, almost all of the scores had significantly changed, including the Functional Assessment of Chronic Illness Therapy-Spiritual Well-Being (FACIT-Sp). The authors concluded that, even though the differences in the primary endpoint were not significant, overall findings supported the use of EPC for advanced cancer patients. In two studies by Bakitas et al., the EPC intervention consisted in a nurse-guided patient education process. In the 2009 study, a positive effect on QoL and depression was reported after 1 year [26]. In the 2015 work, EPC led to an advantage in 1year survival but no significant advantages in primary patientreported outcomes [27]. Another cluster randomized trial conducted by McCorkle et al. did not observe an effect of EPC on patient-reported outcomes [28]. In a recent Danish randomized clinical trial (RCT) [13], patients were randomized to receive early specialist palliative care vs. standard care EPC did not have an impact on the primary outcome of change in primary needs or in QoL items and survival. Undertreatment in the intervention group and crossover/compensation in the control group were hypothesized as potential reasons for these results. The most recent reviews on EPC trials conclude that the quality of studies needs to be improved and that not all outcomes examined to date have been significantly impacted [7, 29].

Three meta-analyses were recently published on EPC, each reporting contrasting results [30-32]. In their review on 43 RCTs with 12,731 patients (including non-oncological), Kavalieratos et al. observed that EPC was associated with improvements in QoL and symptom burden after 1-3 months. No significant advantages were seen for caregivers and there was no evidence of a correlation with longer survival. The results from five studies with a low risk of bias, albeit less consistent, remained significant for QoL but only showed a trend for symptom burden. Palliative care was consistently associated with improvements in advanced care planning, patient and caregiver satisfaction, and lower use of healthcare resources. Evidence of a correlation with other outcomes was mixed.

In 2017, a Cochrane collaboration performed a metaanalysis of EPC studies on cancer patients [31]. Eligibility criteria were fairly strict, the authors retaining only 2 studies with a total of 1614 participants. The review also focused on 15 systematic and qualitative reviews. Primary endpoints of the meta-analysis were health-related QoL, symptom intensity, depression, and survival. EPC significantly improved QoL and symptom burden with respect to standard care. The authors concluded that, although they found only small effect sizes that were clinically relevant in patients with advanced disease and a limited prognosis, the analysis did not reveal a significant effect of EPC on survival or depression.

Another recent meta-analysis assessed the impact of EPC on quality of life in 12 papers with 2454 patients $(72 \%$ of 
Table 1 Baseline characteristics of study participants $(N=179)$

\begin{tabular}{|c|c|c|c|}
\hline & $\begin{array}{l}\text { Standard arm }(N=89) \\
N(\%)\end{array}$ & $\begin{array}{l}\text { Interventional arm }(N=90) \\
N(\%)\end{array}$ & $p$ value $^{\mathrm{f}}$ \\
\hline Median age, years (range) & $69(34-89)$ & $70(36-84)$ & 0.504 \\
\hline \multicolumn{4}{|l|}{ Gender } \\
\hline Male & $62(69.7)$ & $60(66.7)$ & \\
\hline Female & $27(30.3)$ & $30(33.3)$ & 0.668 \\
\hline \multicolumn{4}{|l|}{ Marital status } \\
\hline Married & $60(77.9)$ & $63(82.9)$ & \\
\hline Single & $5(6.5)$ & $5(6.5)$ & \\
\hline Divorced or separated & $3(3.9)$ & $4(5.3)$ & \\
\hline Widowed & $9(11.7)$ & $4(5.3)$ & \\
\hline Unknown/missing & 12 & 14 & 0.276 \\
\hline \multicolumn{4}{|l|}{ ECOG performance status } \\
\hline 0 & $39(43.8)$ & $47(52.2)$ & \\
\hline 1 & $46(51.7)$ & $37(41.1)$ & \\
\hline 2 & $4(4.5)$ & $6(6.7)$ & 0.486 \\
\hline \multicolumn{4}{|l|}{ Assessment of mood symptoms ${ }^{\mathrm{a}}$} \\
\hline \multicolumn{4}{|l|}{ HADS Anxiety subscale } \\
\hline Normal $(\leq 7)$ & $55(64.7)$ & $54(63.5)$ & \\
\hline Borderline abnormal (8-10) & $11(12.9)$ & $18(21.2)$ & \\
\hline Abnormal $(\geq 11)$ & $19(22.4)$ & $13(15.3)$ & \\
\hline Missing & 4 & 5 & 0.628 \\
\hline \multicolumn{4}{|l|}{ HADS Depression subscale } \\
\hline Normal $(\leq 7)$ & $62(72.9)$ & $58(68.2)$ & \\
\hline Borderline abnormal (8-10) & $14(16.5)$ & $17(20.0)$ & \\
\hline Abnormal ( $\geq 11)$ & $9(10.6)$ & $10(11.8)$ & \\
\hline Missing & 4 & 5 & 0.575 \\
\hline \multicolumn{4}{|l|}{ HADS Anxiety subscale } \\
\hline Normal $(\leq 7)$ & $55(64.7)$ & $54(63.5)$ & \\
\hline Abnormal (>7) & $30(35.3)$ & $31(36.5)$ & 0.873 \\
\hline \multicolumn{4}{|l|}{ HADS Depression subscale } \\
\hline Normal $(\leq 7)$ & $62(72.9)$ & $58(68.2)$ & \\
\hline Abnormal (> 7) & $23(27.1)$ & $27(31.8)$ & 0.502 \\
\hline Scores on quality-of-life measures & Means (SD) & Means (SD) & $p$ value $^{\mathrm{g}}$ \\
\hline FACT-G score ${ }^{\mathrm{b}}$ & $69.24(14.56)$ & $70.42(16.08)$ & 0.618 \\
\hline FACT-Ga score ${ }^{c}$ & $121.19(24.28)$ & $121.98(28.20)$ & 0.846 \\
\hline GaCS score ${ }^{\mathrm{d}}$ & $55.19(12.78)$ & $56.31(12.58)$ & 0.612 \\
\hline TOI score ${ }^{\mathrm{e}}$ & $85.87(20.36)$ & $86.29(22.93)$ & 0.900 \\
\hline
\end{tabular}

ECOG Eastern Cooperative Oncology Group, SD standard deviation

${ }^{a}$ The HADS (Hospital Anxiety and Depression Scale consists of two subscales (one for symptoms of anxiety and one for symptoms of depression. Subscale scores range from 0 to $21(0=$ no distress, $21=$ maximum distress); a score $\geq 7$ indicates clinically meaningful anxiety or depression

${ }^{\mathrm{b}}$ FACT-G (Functional Assessment of Cancer Therapy-General) scores range from 0 to 108 (higher scores indicates fewer symptoms: the higher the score, the better the quality of life)

${ }^{\mathrm{c}}$ FACT-Ga (Functional Assessment of Cancer Therapy-Gastric) scores range from 0 to 184

${ }^{\mathrm{d}} \mathrm{GaCS}$ (Gastric Cancer Subscale) scores range from 0 to 76

${ }^{\mathrm{e}}$ TOI (Trial Outcome Index) scores range from 0 to 132

${ }^{\mathrm{f}}$ Chi-squared test or Fisher's exact test

${ }^{g}$ Student's $t$ test for independent samples 
Table 2 Mean change in quality of life scores from T0 to T1

Mean change from T0 to T1

Standard arm (mean, SD, SE)

Interventional arm (mean, SD, SE)

Difference between interventional

$p$ value and standard arm $(95 \% \mathrm{CI})$

\begin{tabular}{lrr}
\hline FACT-G score & $-2.55(11.24 ; 1.39)$ & $-1.64(13.76 ; 1.70)$ \\
FACT-Ga score & $-1.39(22.23 ; 2.77)$ & $1.77(25.24 ; 3.11)$ \\
GaCS score & $0.91(14.14 ; 1.77)$ & $3.19(15.25 ; 1.86)$ \\
TOI score & $-1.30(20.01 ; 2.50)$ & $1.65(22.38 ; 2.76)$
\end{tabular}

$\begin{array}{ll}0.92(-3.43-5.27) & 0.677 \\ 3.16(-5.10-11.43) & 0.450 \\ 2.29(-2.80-7.38) & 0.375 \\ 2.95(-4.43-10.32) & 0.430\end{array}$

$T 0$ date of randomization, $T 112 \pm 3$ weeks after T0, $S D$ standard deviation, $S E$ standard error, $C I$ confidence interval, FACT-G Functional Assessment of Cancer Therapy-General, FACT-Ga Functional Assessment of Cancer Therapy-Gastric, GaCS Gastric Cancer Subscale, TOI Trial Outcome Index

whom had cancer). Overall, a modest effect was seen in favor of specialist palliative care (standardized mean difference 0.16 , 95\% CI 0.01 to 0.31) [32]. In 2017, Ferrel et al. published guidelines on EPC in an American Society of Clinical Oncology Clinical Practice Guideline Update [9]. The same authors had previously published a systematic review of new studies, meta-analyses, and secondary analyses of RCTs that formed the basis for the Provisional Clinical ASCO Opinion of 2012 [33]. The paper comprised six recommendations, each focusing on a clinical issue. The "global direction" of the recommendations was in favor of the early integration of PC into the oncological mainstream. The paper recommended that dedicated outpatient and inpatient EPC services, preferably managed by multidisciplinary teams, be part of the overall cancer healthcare approach. Although an ideal "inception cohort" has still not been universally identified, at least two methods for identifying such a group have been proposed in the literature. The first is a multiparametric score with a cut-off, beyond which a patient is referred for EPC [34]. The second consists in the identification of a number of major and minor indicators, the presence of even just one of the former indicating the need for EPC [35]. Although 13 major indicators of integration between PC and medical oncology have been identified [4], there is still no universal agreement about what "early" means [36]. Automatic referral vs. clinical referral vs. automatic-clinical referral has also been proposed [37].
We recently published the results from an Italian trial comparing systematic (experimental) and on-demand (standard) EPC in 207 pancreatic cancer patients [15, 16]. Findings were substantially in favor of the experimental arm, including the primary aim of the study, QoL, which was found to be improved after 12 weeks. Our data on gastric cancer EPC are less impressive than those on pancreatic cancer.

The study has several limitations that could account for the nonsignificant results. First, a single PC physician rather than a full PC team was responsible for the experimental palliative care intervention. Thus, our palliative care intervention could be defined as rather weak. Secondly, palliative care consultations were standardized, as proposed by Temel et al. [17], but we did not check to make sure that the consultations had been conducted as per Temel's guidelines. Thirdly, any proposals put forward by the palliative care physician had to be discussed with and agreed upon by the primary care oncologist. The gastric cancer study also had a number of other specific weaknesses. In the interventional arm, three of the four QoL scores, including the principal index (TOI), were oriented in a positive sense, while in the control arm, three of the four scores (including TOI) were oriented in a negative sense. However, the difference was not statistically significant. Another potential limitation is that some of the centers taking part were small community hospitals unaccustomed to conducting out clinical research into palliative care. In

Table 3 Quality of life outcomes at T1 (12 \pm 3 weeks)

\begin{tabular}{|c|c|c|c|c|c|}
\hline & Standard arm $(N=65)$ & Interventional arm $(N=66)$ & $\begin{array}{l}\text { Difference between interventional } \\
\text { and standard arm }(95 \% \mathrm{CI})\end{array}$ & & \\
\hline & Mean values (SD) & & & $p$ value $^{\mathrm{a}}$ & Effect size \\
\hline FACT-G score & $67.83(13.74)$ & $71.24(14.25)$ & $3.41(-1.45$ to 8.27$)$ & 0.167 & 0.24 \\
\hline FACT-Ga score & $123.02(24.72)$ & $127.65(24.51)$ & $4.64-3.91$ to 13.18 ) & 0.285 & 0.19 \\
\hline GaCS score & $55.19(12.79)$ & $56.41(12.65)$ & $1.22(-3.19$ to 5.64$)$ & 0.585 & 0.10 \\
\hline TOI score & $88.12(20.51)$ & $91.03(21.14)$ & $2.91(-4.33$ to 10.14$)$ & 0.428 & 0.14 \\
\hline
\end{tabular}

$C I$ confidence interval, SD standard deviation, FACT-G Functional Assessment of Cancer Therapy-General, FACT-Ga Functional Assessment of Cancer Therapy-Gastric, GaCS Gastric Cancer Subscale, TOI Trial Outcome Index

${ }^{\text {a }}$ Adjusted for baseline scores 


\begin{tabular}{|c|c|c|c|}
\hline & Standard arm $(N=77) N(\%)$ & Interventional arm $(N=71) N(\%)$ & $p$ value $^{\mathrm{a}}$ \\
\hline \multicolumn{4}{|l|}{ Chemotherapy administration } \\
\hline Any chemotherapy $\leq 14$ days before death & $6(7.8)$ & $5(7.0)$ & 0.906 \\
\hline Any chemotherapy $\leq 30$ days before death & $9(11.8)$ & $11(15.9)$ & 0.166 \\
\hline \multicolumn{4}{|l|}{ Hospitalization } \\
\hline Any admission $\leq 30$ days before death & $37(49.3)$ & $37(53.6)$ & 0.537 \\
\hline \multicolumn{4}{|l|}{ Emergency Department (ED) visits } \\
\hline Any ED visit $\leq 30$ days before death & $23(32.4)$ & $16(23.9)$ & 0.584 \\
\hline \multicolumn{4}{|l|}{ Place of death } \\
\hline Home or hospice & $50(66.7)$ & $47(70.1)$ & \\
\hline Hospital or nursing home & $25(33.3)$ & $20(29.9)$ & 0.577 \\
\hline \multicolumn{4}{|l|}{ Hospice services } \\
\hline No admission to hospice & $50(64.9)$ & $46(64.8)$ & 0.799 \\
\hline Admission to hospice $\leq 7$ days before death & $7(9.1)$ & $11(15.5)$ & \\
\hline \multirow[t]{2}{*}{ Admission to hospice 7 days before death } & $20(26.0)$ & $14(19.7)$ & \\
\hline & Median value (range) & Median value (range) & \\
\hline \multicolumn{4}{|l|}{ Admission to hospice before death } \\
\hline Number of total admissions & $1(0-2)$ & $1(0-3)$ & 0.517 \\
\hline Days in hospice & $15(1-66)$ & $8(1-42)$ & 0.058 \\
\hline \multicolumn{4}{|c|}{ Admission to palliative care program (home palliative care } \\
\hline Days on palliative care program & $23(2-289)$ & $30(1-190)$ & 0.838 \\
\hline
\end{tabular}

${ }^{a}$ Adjusted for age, gender, marital status, and performance status

addition, patient awareness about prognosis is somewhat limited and unreasonable confidence in the effects of chemotherapy is often high in EoL situations, making this phase of the disease more difficult to manage. Finally, our study was randomized but not blinded.

A number of hypotheses can be put forward to explain our negative results. For example, evaluating the intervention after 12 weeks may be too early for gastric cancer. In fact, Temel [24] and Zimmermann [25] reported more evident effects after 24 weeks. Moreover, Temel observed that results differed from cancer to cancer, with a higher benefit seen for lung cancer with respect to gastrointestinal tumors [24].

PC referral guided by histology, level of disease progression, or intensity of PC needs would seem to be more appropriate than referral based on prognosis or functional status $[36,37]$. Moreover, QoL scores at T0 in the gastric cancer group were better than those of pancreatic cancer patients in both interventional and control arms. Overall, our data suggest that gastric cancer was less symptomatic than pancreatic cancer in the respective studies when accrual began. The mean change in TOI from $\mathrm{T} 0$ to $\mathrm{T} 1$ in the conventional arm was -1.30 for gastric patients and -4.47 for pancreatic patients, while that of disease-specific issues was +0.91 for gastric cancer and -2.23 for pancreatic cancer, indicating a less dramatic change in the conventional arm of the gastric study. Moreover, median OS was 8.3 months in the pancreatic cancer population compared to 9.9 months in the gastric cancer cohort and a moderately high percentage of patients $(43 \%)$ in the standard arm received early palliative care. Looking at our data on basic QoL, symptom burden, and OS, we can conclude that gastric patients showed a different statistical trend to that of the pancreatic population, were less advanced in terms of life expectancy, and had a lower symptom burden and a less impacted QoL. Thus, evaluation after 12 weeks may have been too early as the effect of EPC on the worst clinical situations was "diluted" due to the fact that patients were still not heavily symptomatic. Finally, as both studies were activated at the same time but gastric cancer patient accrual was slower, we can hypothesize that the oncologists in the conventional arm had the opportunity of going through a sort of "hands-on education" that enabled them to manage the last patients recruited in the gastric study in a "more palliative way".

In conclusion, there is increasing evidence of the efficacy of EPC. Although some studies have published negative results, nonsignificant data often show a trend in favor of the experimental EPC arm. Overall, our findings indicate that the following items require special attention when conducting research into EPC: type of EPC intervention (monoprofessional vs. multiprofessional), risk of contamination and/or crossover with the conventional arm, study duration, timing of evaluation, timeliness of cohort inception (this will probably differ for different cancer types), level of symptom burden, and reduced QoL of recruited patients. 
Acknowledgements The authors thank the following members of the Early Palliative Care Italian Study Group and co-authors of the paper for their technical help: Flavia Pagan (IRST IRCCS, Meldola), Vincenzo Dadduzio (Veneto Institute of Oncology IOV-IRCCS, Padus), Tiziana Trapasso (SS Trinità Hospital, Sora, ASL Frosinone), Camilla Di Nunzio (Guglielmo da Saliceto Hospital, Piacenza), Paolo Pedrazzoli (Fondazione IRCCS Policlinico San Matteo, Pavia), Cristina Autelitano (Arcispedale S. Maria Nuova-IRCCS, Reggio Emilia), Alessandro Comandone (Presidio Humanitas Gradenigo, Turin), Carla Codecà (San Paolo Hospital, Milan), Francesca Martella (Azienda USL Toscana Centro, S. Maria Annunziata Hospital, Florence), Angela Buonadonna (Aviano National Cancer Institute, Aviano), Laura Toppo (Azienda Socio Sanitaria Territoriale, Cremona), Augusto Caraceni (Fondazione IRCCS Istituto Nazionale dei Tumori, Milan), Giovanni Luchena (Sant'Anna Hospital, Asst-Lariana, Como), Maria Teresa Cattaneo (L. Sacco Hospital, Milan), Massimo Luzzani (E.O. Galliera Hospitals, Genoa), Roberta Gauna (Ospedale degli Infermi, Ponderano), and Cristina Pittureri (AUSL Romagna, Cesena). The authors also thank Grainne Tierney and Cristiano Verna for editorial assistance.

Authors' contribution Conception and design: Marco Maltoni, Emanuela Scarpi, Chiara Maria Broglia, and Oriana Nanni. Collection and assembly of data: Monia Dall'Agata, Vittorina Zagonel, Teresa Gamucci, Raffaella Bertè, Elisabetta Sansoni, Elena Amaducci, Chiara Maria Broglia, Sara Alquati, Ferdinando Garetto, Stefania Schiavon, Silvia Quadrini, Elena Orlandi, Andrea Casadei Gardini, Silvia Ruscelli, Daris Ferrari, Maria Simona Pino, Roberto Bortolussi, Federica Negri, Silvia Stragliotto, Filomena Narducci, Martina Valgiusti, Alberto Farolfi, Oriana Nanni, Romina Rossi, and Marco Maltoni. Data analysis and interpretation: Marco Maltoni and Emanuela Scarpi. Manuscript writing: All authors. Final approval of manuscript: All authors and members of the Early Palliative Care Italian Study Group.

Funding information This trial was partially supported by a research grant from the Italian Ministry of Health (RF-2011-02350971).

\section{Compliance with ethical standards}

The study was approved by the Ethics Committee of each participating center and was performed in accordance with the principles laid down in the 1964 Declaration of Helsinki and its later amendments.

Conflict of interest The authors declare that they have no conflict of interest.

Informed consent Written informed consent was obtained from all individuals taking part in the study.

\section{References}

1. World Health Organization. WHO definition of palliative care. http://who.int/cancer/palliative/definition/en/. Accessed 31st January, 2018

2. Hui D, Bruera $E$ (2016) Integrating palliative care into the trajectory of cancer care. Nat Rev Clin Oncol 13:159-171

3. Zagonel V, Torta R, Franciosi V, Brunello A, Biasco G, Cattaneo D, Cavanna L, Corsi D, Farina G, Fioretto L, Gamucci T, Lanzetta G, Magarotto R, Maltoni M, Mastromauro C, Melotti B, Meriggi F, Pavese I, Piva E, Sacco C, Tonini G, Trentin L, Ermacora P, Varetto A, Merlin F, Gori S, Cascinu S, Pinto C, AIOM Simultaneous \& Continuous Care (SCC) Task Force-ESMO-DCs (2016) Early integration of palliative care in oncology practice: results of the Italian
Association of Medical Oncology (AIOM) survey. J Cancer 7: 1968-1978

4. Hui D, Bansal S, Strasser F, Morita T, Caraceni A, Davis M, Cherny N, Kaasa S, Currow D, Abernethy A, Nekolaichuk C, Bruera E (2015) Indicators of integration of oncology and palliative care programs: an international consensus. Ann Oncol 26:1953-1959

5. Maltoni M, Scarpi E, Pittureri C, Martini F, Montanari L, Amaducci E, Derni S, Fabbri L, Rosati M, Amadori D, Nanni O (2012) Prospective comparison of prognostic scores in palliative care cancer populations. Oncologist 17:446-454

6. Gómez-Batiste X, Martínez-Muñoz M, Blay C, Amblàs J, Vila L, Costa X, Espaulella J, Vallanueva A, Oller R, Martori JC, Constante C (2017) Utility of the NECPAL CCOMS-ICO tool and the surprise question as screening tools for early palliative care and to predict mortality in patients with advanced chronic conditions: a cohort study. Palliat Med 31:754-763

7. Davis MP, Temel JS, Balboni T, Glare P (2015) A review of the trials which examine early integration of outpatient and home palliative care for patients with serious illnesses. Ann Palliat Med 4: 99-121

8. Cherny N, Catane R, Schrijvers D, Kloke M, Strasser F (2010) European Society for Medical Oncology (ESMO) program for the integration of oncology and palliative care: a 5-year review of the designated centers' incentive program. Ann Oncol 21:362-369

9. Ferrell BR, Temel JS, Temin S, Alesi ER, Balboni TA, Basch EM, Firn JI, Paice JA, Peppercorn JM, Phillips T, Stovall EL, Zimmermann C, Smith TJ (2017) Integration of palliative care into standard oncology care: American Society of Clinical Oncology clinical practice guideline update. J Clin Oncol 35:96-112

10. Quill TE, Abernethy AP (2013) Generalist plus specialist palliative care — creating a more sustainable model. N Engl J Med 368:11731175

11. Hui D, Bruera E (2015) Models of integration of oncology and palliative care. Ann Palliat Med 4:89-98

12. Rabow MW, Dibble SL, Pantilat SZ, McPhee SJ (2004) The comprehensive care team: a controlled trial of outpatient palliative medicine consultation. Arch Intern Med 164:83-91

13. Groenvold M, Petersen MA, Damkier A, Neergaard MA, Nielsen JB, Pedersen L, Sjøgren P, Strömgren AS, Vejlgaard TB, Gluud C, Lindschou J, Fayers P, Higginson IJ, Johnsen AT (2017) Randomised clinical trial of early specialist palliative care plus standard care versus standard care alone in patients with advanced cancer: the Danish Palliative Care Trial. Palliat Med 31:814-824

14. Parikh RB, Kirch RA, Smith TJ, Temel JS (2013) Early specialty palliative care - translating data in oncology into practice. N Engl J Med 369:2347-2351

15. Maltoni M, Scarpi E, Dall'Agata M, Zagonel V, Bertè R, Ferrari D, Broglia CM, Bortolussi R, Trentin L, Valgiusti M, Pini S, Farolfi A, Casadei Gardini A, Nanni O, Amadori D, Early Palliative Care Italian Study Group (EPCISG) (2016) Systematic versus ondemand early palliative care: results from a multicentre, randomised clinical trial. Eur J Cancer 65:61-68

16. Maltoni $\mathrm{M}$, Scarpi $\mathrm{E}$, Dall'Agata $\mathrm{M}$, Schiavon $\mathrm{S}$, Biasini $\mathrm{C}$, Codecà C, Broglia CM, Sansoni E, Bortolussi R, Garetto F, Fioretto L, Cattaneo MT, Giacobino A, Luzzani M, Luchena G, Alquati S, Quadrini S, Zagonel V, Cavanna L, Ferrari D, Pedrazzoli P, Frassineti GL, Galiano A, Casadei Gardini A, Monti M, Nanni O, Early Palliative Care Italian Study Group (EPCISG) (2016) Systematic versus on-demand early palliative care: a randomised clinical trial assessing quality of care and treatment aggressiveness near the end of life. Eur J Cancer 69:110-118

17. Temel JS, Greer JA, Muzikansky A, Gallagher ER, Admane S, Jackson VA, Dahlin CM, Blinderman CD, Jacobsen J, Pirl WF, Billings JA, Lynch TJ (2010) Early palliative care for patients with metastatic non-small-cell lung cancer. N Engl J Med 363:733-742 
18. National Consensus Project (2009) Clinical practice guidelines for quality palliative care. 2nd ed. Pittsburgh. http:// nationalconsensusproject.org. Accessed 31st January 2018

19. Cella DF, Tulsky DS, Gray G, Sarafian B, Linn E, Bonomi A, Silberman M, Yellen SB, Winicour P, Brannon J (1993) The Functional Assessment of Cancer Therapy scale: development and validation of the general measure. J Clin Oncol 11:570-579

20. Zigmond AS, Snaith RP (1983) The hospital anxiety and depression scale. Acta Psychiatr Scand 67:361-370

21. Chattat R, Ottoboni G, Zeneli A, Berardi MA, Cossu V, Maltoni M (2016) The Italian version of the FAMCARE scale: a validation study. Support Care Cancer 24:3821-3830

22. Medigovich K, Porock D, Kristjanson LJ, Smith M (1999) Predictors of family satisfaction with an Australian palliative home care service: a test of discrepancy theory. J Palliat Care 15:48-56

23. Schafer JL (1997) Analysis of incomplete multivariate data. Chapman and Hall, London

24. Temel JS, Greer JA, El-Jawahri A, Pirl WF, Park ER, Jackson VA, Back AL, Kamdar M, Jacobsen J, Chittenden EH, Rinaldi SP, Gallagher ER, Eusebio JR, Li Z, Muzikansky A, Ryan DP (2017) Effects of early integrated palliative care in patients with lung and GI cancer: a randomized clinical trial. J Clin Oncol 35:834-841

25. Zimmermann C, Swami N, Krzyzanowska M, Hannon B, Leighl N, Oza A, Moore M, Rydall A, Rodin G, Tannock I, Donner A, Lo C (2014) Early palliative care for patients with advanced cancer: a cluster-randomised controlled trial. Lancet 383:1721-1730

26. Bakitas M, Lyons KD, Hegel MT, Balan S, Brokaw FC, Seville J, Hull JG, Li Z, Tosteson TD, Byock IR, Ahles TA (2009) Effects of a palliative care intervention on clinical outcomes in patients with advanced cancer: the Project ENABLE II randomized controlled trial. JAMA 302:741-749

27. Bakitas MA, Tosteson TD, Li Z, Lyons KD, Hull JG, Li Z, DionneOdom JN, Frost J, Dragnev KH, Hegel MT, Azuero A, Ahles TA (2015) Early versus delayed initiation of concurrent palliative oncology care: patient outcomes in the ENABLE III randomized controlled trial. J Clin Oncol 33:1438-1445

28. McCorkle R, Jeon S, Ercolano E, Lazenby M, Reid A, Davies M, Viveiros D, Gettinger S (2015) An advanced practice nurse coordinated multidisciplinary intervention for patients with late-stage cancer: a cluster randomized trial. J Palliat Med 18:962-969
29. Tassinari D, Drudi F, Monterubbianesi MC, Stocchi L, Ferioli I, Marzaloni A, Tamburini E, Sartori S (2016) Early palliative care in advanced oncologic and non-oncologic chronic diseases. A systematic review of the literature. Rev Recent Clin Trials 11:63-71

30. Kavalieratos D, Corbelli J, Zhang D, Dionne-Odom JN, Ernecoff NC, Hanmer J, Hoydich ZP, Ikejiani DZ, Klein-Fedyshin M, Zimmermann C, Morton SC, Arnold RM, Heller L, Schenker Y (2016) Association between palliative care and patient and caregiver outcomes: a systematic review and meta-analysis. JAMA 316: 2104-2114

31. Haun MW, Estel S, Rücker G, Friederich HC, Villalobos M, Thomas M, Hartmann M (2017) Early palliative care for adults with advanced cancer. Cochrane Database Syst Rev 12(6):CD011129

32. Gaertner J, Siemens W, Meerpohl JJ, Antes G, Meffert C, Xander C, Stock S, Mueller D, Schwarzer G, Becker G (2017) Effect of specialist palliative care services on quality of life in adults with advanced incurable illness in hospital, hospice, or community settings: systematic review and meta-analysis. BMJ 357:j2925

33. Smith TJ, Temin S, Alesi ER, Abernethy AP, Balboni TA, Basch EM, Ferrell BR, Loscalzo M, Meier DE, Paice JA, Peppercorn JM, Somerfield M, Stovall E, Von Roenn JH (2012) American Society of Clinical Oncology provisional clinical opinion: the integration of palliative care into standard oncology care. J Clin Oncol 30:880 887

34. Glare P, Plakovic K, Schloms A, Egan B, Epstein AS, Kelsen D, Saltz L (2013) Study using the NCCN guidelines for palliative care to screen patients for palliative care needs and referral to palliative care specialists. J Natl Compr Cancer Netw 11:1087-1096

35. Hui D, Mori M, Watanabe SM, Caraceni A, Strasser F, Saarto T, Cherny N, Glare P, Kaasa S, Bruera E (2016) Referral criteria for outpatient specialty palliative cancer care: an international consensus. Lancet Oncol 17:e552-e559

36. Gaertner J, Wolf J, Hallek M, Glossmann JP, Voltz R (2011) Standardizing integration of palliative care into comprehensive cancer therapy - a disease specific approach. Support Care Cancer 19: $1037-1043$

37. Hui D, Mori M, Meng YC, Watanabe SM, Caraceni A, Strasser F, Saarto T, Cherny N, Glare P, Kaasa S, Bruera E (2018) Automatic referral to standardize palliative care access: an international Delphi survey. Support Care Cancer 26:175-180 